\title{
Complex Systems and Health Behavior Change: Insights from Cognitive Science
}

\author{
Mark G. Orr, PhD; David C. Plaut, PhD
}

Objective: To provide proof-of-concept that quantum health behavior can be instantiated as a computational model that is informed by cognitive science, the Theory of Reasoned Action, and quantum health behavior theory. Methods: We conducted a synthetic review of the intersection of quantum health behavior change and cognitive science. We conducted simulations, using a computational model of quantum health behavior (a constraint satisfaction artificial neural network) and tested whether the model exhibited quantum-like behavior. Results: The model exhibited clear signs of quantum-like behavior. Conclusions: Quantum health behavior can be conceptualized as constraint satisfaction: a mitigation between current behavioral state and the social contexts in which it operates. We outlined implications for moving forward with computational models of both quantum health behavior and health behavior in general.

Key words: health behavior, behavior change, quantum change, complex systems, cognitive science, computational modeling, agent-based modeling

Am J Health Behav. 2014;38(3):404-413

DOI: http://dx.doi.org/ 10.5993/AJHB.38.3.9
$\mathrm{T}$ The quantum behavior change paradigm considers the possibility that health behavior may be governed by dynamic non-linear and chaotic processes in addition to, or instead of, more rational, linear processes. ${ }^{1}$ This idea is central to work on "catastrophic" pathways to health behavior change, where behavior change is characterized by sudden and dramatic shifts. ${ }^{2}$

In 2008, Resnicow and Page ${ }^{3}$ put forth a novel analysis of the quantum behavior change paradigm that generated several implications for health behavior theory and practice. The dominant implication was that to understand quantum behavior change we may need to integrate the complex systems approach with current theory. This approach is useful, if not essential, when one is trying to understand the behavior of a system that has many components that dynamically interact with one another. A prototypal example of a complex system is a flock of birds. Although the "rules" that dictate the behavior of each individual bird may be understood, it is difficult to extrapolate from these rules to an understanding of the dynamics of the system as a whole without some sort of model. By necessity, such models are almost exclusively in

Mark G. Orr, Associate Research Scientist, Columbia University, Department of Epidemiology, Mailman School of Public Health, New York, NY. David C. Plaut, Professor, Carnegie Mellon University, Department of Psychology, Pittsburgh, PA. Correspondence Dr Orr; mo2259@columbia.edu computational form and require computer simulation for understanding how the dynamics of the system unfold.

Resnicow and Page $^{3}$ provided a description of what a complex systems model of quantum health behavior change might look like-behavior at any point in time was dictated by the arrangement of ping-pong balls, serving as mental constructs that were stuck to the surface of a rotating, circular drum-like lottery machine. Although this analogy captured the essence of a complex system and served well to illustrate a point, it was not implemented in a computational format and, more importantly, it was not informed by the literature on computational modeling in cognitive science--a field that specializes in computational models of dynamic mental processes. ${ }^{4}$

In this paper we present a theory of quantum health behavior that is grounded in cognitive science and complex systems and judged via computer simulation to what extent it exhibits quantum behavior. To stay grounded in health behavior theory, we do this in the context of the reasoned action approach, ${ }^{5}$ using the Theory of Reasoned Action $^{6}$ as the simplest case for purposes of illustration.

Our primary goal is to provide proof-of-concept that quantum health behavior theory can be conceputalized in terms of cognitive science, health behavior theory, and complex systems simultaneously. In essence we are presenting a cognitive sci- 
ence approach to health behavior and so discuss the implications beyond the specifics of the quantum health behavior paradigm to health behavior theory, in general. In the end, we hope to spur interest in the potential for a new sub-discipline, what we provisionally call the computational heath behavior modeling approach.

\section{Cognitive Science, Computational Modeling and Behavior Change}

Cognitive science is the study of the mind as an information-processing system. Borrowing heavily from computer science, psychology, anthropology, artificial intelligence, linguistics, philosophy and neuroscience, this discipline has yield benefit over the past 60 years. ${ }^{7,8}$

Computational modeling and simulation have been central theoretical and methodological tools in cognitive science from its inception. The earliest models in the 1950s focused on chess and general symbolic logic problems. ${ }^{9}$ Today, in addition to more basic cognitive processes, computational modeling is used to model social processes, ${ }^{10}$ attitude formation, ${ }^{11}$ and health behavior. ${ }^{12}$

In cognitive science, computational modeling is used extensively because it elucidates cognitive processes in explicit algorithmic terms that define how information is processed, both in terms of the structures used to store, transform and pass-on information, and the nature of the information itself. In short, an algorithm defines how input becomes output.

There is a direct analog between computational modeling in cognitive science and the complex systems approach to studying health behavior at the population level. The computational model, in both cases, is a dynamic representation of the key processes that are supposed to explain the behavior under investigation. It yields insight into important population health phenomena ${ }^{13}$ as well as cognitive phenomena ${ }^{14}$ precisely because it deals with the complexity as it is, and affords a glimpse into the dynamics of the system, both of which are more difficult when using other approaches. Agent-based modeling is an excellent example in population health. Agents representing people, interact in various ways with their environment, and with each other, to produce patterns of populationlevel health behavior. The fundamental difference here is only one of scale: cognitive science builds models of individuals instead of populations, in which the interacting subcomponents correspond to mental constructs instead of people.

The link between cognitive science and health behavior theory is clear once we recognize that the core components of health behavior change theory are rooted in the notion of information-processing. Mental constructs prominent in health behavior theories, eg, beliefs, attitudes, expectations, selfefficacy, norms, and social perception/interaction are constructed from, and processed as, information about the world. This idea is mirrored in the fields of social cognition and social cognitive neuroscience where the assumption is the same, ie, social behavior is dependent on information processing mechanisms and constructs.

\section{A Computational Model of Quantum Behavior Change}

We are proposing a computational instantiation, as proof-of-concept, of the quantum behavior change theory. To date, no model of quantum health behavior exists aside from the metaphorical model of Resnicow and Page. ${ }^{3}$ Therefore, we derived the model proposed here from our prior work $^{12}$ on the development of a dynamic computational model of the Theory of Reasoned Action (TRA). ${ }^{6}$ We used the TRA instead of the more recent Theory of Planned Behavior (TPB) ${ }^{15}$ or the Integrated Model (IM) ${ }^{16}$ because its relative simplicity was more suited for developing a novel computational model that lacked any prior work from which to draw.

Before we present the details of the model, we will first give an overview of what the model and simulations were designed to represent. This modeling effort tries to capture, in simplified form, the dynamics of health behavior intention amid changing social contexts. Think of the time-course of intention as a person goes through life, at some arbitrary temporal scale, while social contexts change. At every point in this trajectory, our model computes intention as a state that incorporates both the present state of intention and the intentions of those around him/her (the social context).

The data that are generated from the model are analogous to a time-series of one person's experience over many units of time. Although the time steps in our simulations are rather arbitrary, we think of each step in time as a discrete social context where specific processes happen in a particular order, ie, a person enters a new social context with an intention state equal to the ending state from the most recent prior social context, processes the new context to form a new intention state, and then enters a new context.

Next, we present the core assumptions of our model. We drew from several distinct but related research literatures to construct the model's assumptions because the TRA does not provide all of the necessary assumptions to generate a dynamic model of quantum health behavior. It is conventional to state the detailed assumptions of a computational model to show its logic and to provide insight into its empirical grounding. Issues of model validity will be discussed.

\section{General Assumptions of the Model}

Assumption 1: Beliefs alone drive the health behavior of the individual. Our model is an extension of the TRA in which intentions drive behaviors, and are themselves driven by beliefs, through attitudes and norms. The TRA characterizes a person's intention to perform a behavior as a linear 
aggregation across a set of beliefs, each with its own valence with respect to the behavior in question. Positive belief valence contributes to "intending" to do a behavior, negative to "not intending." Our prior modeling work with the TRA established, with empirical validation, that intention can be conceived as a dynamic collection of beliefs alone; intention, attitudes and social norms do not need to be represented directly in the model. ${ }^{12}$

Assumption 2. Social context influences health behavior of the individual directly. From network science we have compelling evidence that people influence others regarding a host of health behaviors, eg, smoking cessation, ${ }^{17}$ drug use, ${ }^{18}$ and obesity ${ }^{13}$ (see ${ }^{19}$ for a review). Our working assumption is that social context can influence what beliefs are active in a person's memory, and thus, affect behavioral intention. Currently, there is a dearth of psychological research on how beliefs change as a function of social context.

Assumption 3. Intention to perform a behavior is a dynamic, on-the-fly process we call intention formation that is driven by constraint satisfaction. Recent advances in the literatures on attitude formation and personality theory have originated, to some degree, from an adoption of the cognitive science computational modeling approach. Here, attitude formation and behavior (from each respective field) are conceptualized as arising, in the moment, from the dynamic activation of memory structures from exposure to social contexts/situations. ${ }^{11,20,21}$ Thus, attitudes and behavior are assumed to be determined, simultaneously, by a person's memory structure and social context/situation. This advance was made possible through what is called constraint satisfaction in cognitive science. ${ }^{22}$

In our model, beliefs and associations among them are the memory structures. Social context, which is the beliefs of others, activates the memory structures via social influence. Intention arises from the dynamic interaction between both the memory structures and social context via constraint satisfaction. In the terminology of complex adaptive systems, intention emerges from the dynamic relations among beliefs in memory and the social context.

More specifically, constraint satisfaction operates over a set of psychological constructs (in our model these are beliefs) defined as computational processing units that interact with each other and with the external social context (in our model, external social context is defined as others' beliefs). Each processing unit represents a hypothesis about whether or not, or how strongly, a belief is activated in a person's memory and is linked to the social context such that belief $X$ of someone in the social context will partially activate believe $X$ in the model.

The concepts of activation and constraint are central to constraint satisfaction. Activation is like the air pressure in a balloon. A belief without ac- tivation is like a balloon with zero air pressurethe balloon is flaccid. A high degree of activation is similar to a balloon with a high level of air pressure-the balloon is large and near its maximum size. The degree of activation is an analog for how strongly a belief is activated in a person's memory. The constraint in constraint satisfaction captures the idea that the activation in one belief is dependent on the activation of other beliefs, either in an excitatory or inhibitory manner. Imagine 2 balloons that are connected by a small air hose. If balloon A inhibits balloon B, then as the air pressure increases in A it decreases in B. If, on the other hand, A excites B, the relation is direct instead of inverse. Furthermore, another person's beliefs (in the social context) have a weak but strictly excitatory constraint on the beliefs in a person's memory. That is, exposure to another's beliefs will excite the analogous beliefs in the model.

This conceptualization of intention as forming on the fly is not in contradiction with the TRA. Theoretical work on the TRA (and the related TPB and IM frameworks) is just beginning to explore the issue of memory structures and access (see ${ }^{23}$ for a thoughtful conceptualization on how to move forward in this area and ${ }^{24}$ for work on probing the belief memory structures in the TRA).

Assumption 4. An individual's intention state from one social context carries over to the next adjacent social context. Constraint satisfaction must take into account any prior activation of the memory structures. That is, under most conditions, the memory structures have some activation before the constraint satisfaction process begins. Given that our simulations attempt to capture intention states over a series of social contexts, the issue of whether the activation from a prior social context is "carried over" is necessary to consider. In our simulations, we assumed that intention states carry over from one social context to the next.

Evidence supports this assumption. First, idiographic and high-density time series studies support that addictive behaviors are partly a function of past behaviors. ${ }^{25,26}$ Second, this assumption has been used in the study of personality and behavior to explain why people act differently in similar contexts separated in time - recent past behaviors seem to affect current behaviors. ${ }^{10,21,27}$ Finally, the assumption that a person is simply "turned-off" between contexts and experiences suffers from a lack of face validity.

\section{Specific Assumptions of the Model}

The general assumptions above provide insight into the higher-level assumptions of the model. In contrast, here we provide the detailed assumptions concerning the structure of the model, and where possible, the rationale for these assumptions. These assumptions were borrowed directly from our previous work on the development of a computational model of the TRA. ${ }^{12}$

Belief structure. In Figure 1, Panel A, we show 


\section{A Constraint Satisfaction Model of Quantum Health Behavior}

A

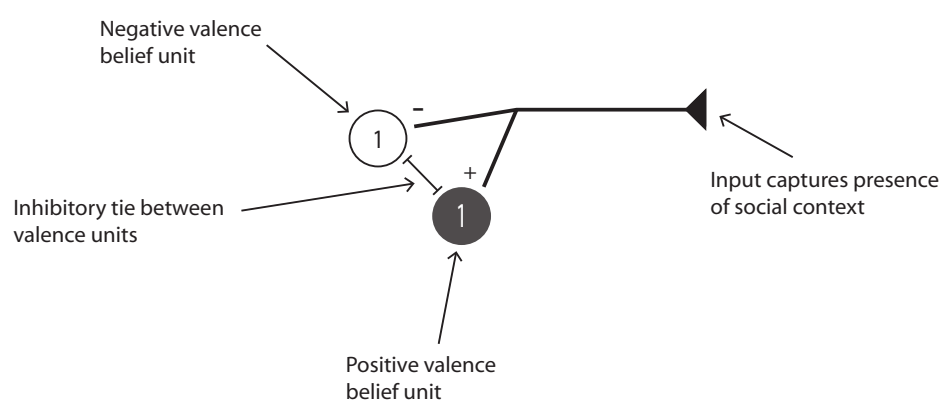

B

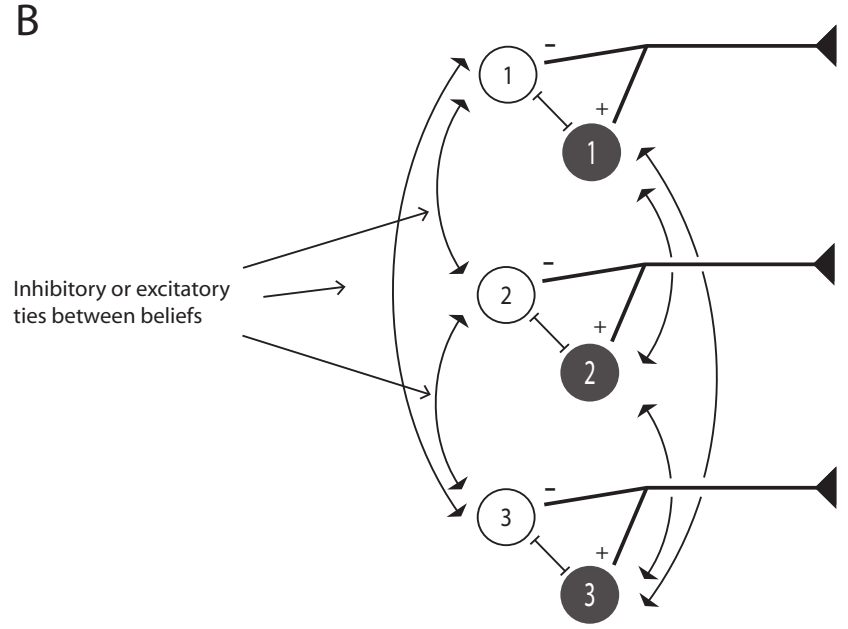

Note.

Panel A represents a single belief; Panel B, a model with 3 beliefs (values in the valence units denote the belief number). See the text for further information.

the structure of a single belief. It is split into 2 separate valence units, one to represent the positive valence of the belief (the black circle), the other to represent the negative valence (the white circle). The connecting tie between the units represents an inhibitory constraint between the valence units-if one is active, it detracts from the activation of the other, eg, if the negative unit is highly active, it takes away from the activation of the positive unit. Despite the between-unit inhibition, the structure of beliefs in our model does allow for the possibility of both valence units to be active simultaneously, ie, beliefs are assumed to be 2-dimensional. A1though this assumption is clearly at odds with the assumption of unidimensional beliefs in the TRA, it allows for the possibility of ambivalent attitudes / intention. This accords with recent advances in the ambivalence of attitudes, ${ }^{28,29}$ and in prior work on developing computational models of cognitive dissonance. ${ }^{30}$ Our intuition was that ambivalence may be a key property of quantum health behavior.

Belief unit activation. Valence unit activitation ranges from 0 to $1 ; 0$ is considered not active; 1 is highly active. At any point in time, the activation of each valence unit is determined by 3 things: its prior activation, the activation of its compliment (the other valence unit), and the presence of others' beliefs (the social context). The presence of others' 


\section{Table 1 \\ The Belief Structure of Each of the Three Social Contexts Used in the Simulations}

\begin{tabular}{|c|c|c|c|c|c|c|c|c|c|c|}
\hline \multirow[b]{2}{*}{ Social Context } & \multicolumn{5}{|c|}{$\begin{array}{c}\text { Positive Valence Units } \\
\text { Belief Number }\end{array}$} & \multicolumn{5}{|c|}{$\begin{array}{l}\text { Negative Valence Units } \\
\text { Belief Number }\end{array}$} \\
\hline & 1 & 2 & 3 & 4 & 5 & 1 & 2 & 3 & 4 & 5 \\
\hline A, Positive/Intend & 1 & 1 & 1 & 1 & 1 & 0 & 0 & 0 & 0 & 0 \\
\hline B, Negative/Not Intend & 0 & 0 & 0 & 0 & 0 & 1 & 1 & 1 & 1 & 1 \\
\hline C, Ambivalent & 1 & 1 & 0 & 0 & 0 & 1 & 1 & 0 & 0 & 0 \\
\hline \multicolumn{11}{|c|}{$\begin{array}{l}\text { Note. } \\
\text { 1. The values in the table represent the activation level of the beliefs in the social context which were presented to the } \\
\text { model during the simulation. } \\
\text { 2. The labels } A, B \text { and } C \text { refer to the context and are referenced in the text and Table } 2 .\end{array}$} \\
\hline
\end{tabular}

beliefs should be thought of as an interaction with a social context.

Global model structure. Panel B (Figure 1) illustrates the structure of the full model, which is simply an expansion of a single belief into a set of beliefs, each one being identical in structure to what is shown in Panel A. The only additional information, compared to Panel A, are the ties (arrows) between each belief. These depict the constraints between beliefs which, in this model, are always fixed to be excitatory. Our model is constructed from 5 beliefs; only 3 are shown in Figure $1 \mathrm{~b}$ to make the illustration clearer.

Behavioral intention. Behavioral intention, in TRA parlance, is determined by the pattern of activation over the whole system. If the positive valence units are more active than the negative units on average, then the system is in a state similar to "intending" to do a behavior. If, on the other hand, the negative units win out, then it is "not intending" to do a behavior. Importantly, our model allows for ambiguous states to arise, in which both positive and negative units are nearly equally active.

\section{METHODS}

The simulation amounted to exposing the model to only 3 social contexts, but many times (200 exactly) and in a random order. Each of the contexts was unique; one was heavily biased to "intend," another to "not intend," and a third was "ambivalent." As the model was exposed to the social contexts, the activation levels of all belief valence units were recorded, yielding a time-series of the intention state of the model.

We ran 20 independent simulations. The only difference between simulations was the ordering of the sequence of social contexts; each was a unique, random order. The details of the simulation methods are presented next.

Structure of the social contexts. It is useful to imagine that each social context is just another model, identical to the model we represent in Figure 1, with the same terminology (valence units and belief number); however, the social context is not, in practice, a model, but just a set of inputs that interact with the model during the simulation.

Table 1 shows the belief structure of each of the 3 unique social contexts. Social context A was strongly intend (all of the positive belief units were highly active; the negative units were not active); context B was strongly not-intend; context C was ambivalent such that, within each belief, the activation levels of both valence units were equated and across beliefs some were highly activated and others were not at all activated.

Exposure of a social context to the model. The interaction between the model and the social context was via a direct mapping between the valence units of both. In other words, the positive valence unit of belief 1 in the social context activated the positive valence unit of belief 1 in the model, and so on, for all valence units in the model. Furthermore, the model only received $50 \%$ of the activation of the social context-eg, if a social context valence unit is 1 , then the model only received a value of 0.5 activation from the social context.

Model dynamics. The initial state of the model was driven entirely by the first social context. For example, in Table 2, the first social context of Simulation 1 was exposure to social context C. After the initial social context, the dynamics were driven by the series of social contexts and the prior activation of the model's belief units (the prior intention state). That is, for all of the simulation, except at the beginning, the state of the model was dependent on both its immediate past intention state and the current social context. Throughout each simulation, the social contexts changed. Table 2 illustrates this within each simulation and how the ordering of social contexts varied across simulations. These orderings are for illustrative purposes 


\section{Table 2 \\ An Illustration of the Sequential Ordering of Social Contexts Across the 20 Simulations}

\begin{tabular}{cccccc}
\hline & \multicolumn{7}{c}{ Simulation Number } \\
\cline { 2 - 6 } Time Step & $\mathbf{1}$ & $\mathbf{2}$ & $\mathbf{3}$ & $\ldots$ & $\mathbf{2 0}$ \\
\hline 1 & $\mathrm{C}$ & $\mathrm{A}$ & $\mathrm{A}$ & $\ldots$ & $\mathrm{B}$ \\
2 & $\mathrm{~B}$ & $\mathrm{C}$ & $\mathrm{B}$ & $\ldots$ & $\mathrm{A}$ \\
3 & $\mathrm{~A}$ & $\mathrm{~B}$ & $\mathrm{C}$ & $\ldots$ & $\mathrm{C}$ \\
4 & $\mathrm{~B}$ & $\mathrm{~B}$ & $\mathrm{~B}$ & $\ldots$ & $\mathrm{A}$ \\
5 & $\mathrm{C}$ & $\mathrm{C}$ & $\mathrm{C}$ & $\ldots$ & $\mathrm{C}$ \\
6 & $\mathrm{~A}$ & $\mathrm{~A}$ & $\mathrm{~A}$ & $\ldots$ & $\mathrm{B}$ \\
$\cdot$ & $\mathrm{A}$ & $\mathrm{B}$ & $\mathrm{B}$ & $\ldots$ & $\mathrm{B}$ \\
& $\mathrm{C}$ & $\mathrm{C}$ & $\mathrm{C}$ & $\ldots$ & $\mathrm{A}$ \\
600 & $\mathrm{~B}$ & $\mathrm{~A}$ & $\mathrm{~A}$ & $\ldots$ & $\mathrm{C}$ \\
& $\mathrm{A}$ & $\mathrm{C}$ & $\mathrm{C}$ & $\ldots$ & $\mathrm{C}$ \\
\hline
\end{tabular}

Note.

The values in the table represent the social context label (see Table 1).

only and do not reflect the exact ordering for each simulation.

\section{RESULTS}

Figure 2, which shows 4 of the 20 simulations, illustrates the key behaviors of the model. There were 4 distinct dynamic profiles. In the first row (panel A and B) the positive valence bank (to "intend") won out early on and maintained a high degree of activity throughout the simulation; the negative valence bank (to "not intend") was pushed to zero activation. The second row illustrates the ability of the model to change rapidly from "intend" to "not intend." Row 3 shows a constant ambivalence towards the behavior. Both valence banks were highly active throughout the simulation. The final row exhibits a mixture of "not intend" and ambivalence.

The goal of this modeling effort was to judge to what extent our computational model exhibited quantum-like behavior. This is a non-trivial task because there is no definitive statistical test for quantum behavior. However, the complex systems literature, to include catastrophe theory, provides several well established qualitative criteria that are applicable to these simulations. ${ }^{31,32}$

Our model displayed some of the key signatures of a complex system. First, it is clear that the statistical signature of the inputs (that $1 / 3$ were favoring intend, $1 / 3$ not intend, and $1 / 3$ ambivalent) did not manifest in time-series of intention for most if not all simulations. Simply put, the output was not linearly related to the input, a classic signature of complex systems. Panel B of Figure 2 exemplifies this behavior as an extreme case, ie, the time-series almost ignored the inputs. Second, Panels B, D, and F of Figure 2 demonstrate path dependence, another signature of complex systems in which a system is attracted to and remains in one behavioral state. Third, Panel C shows bifurcation, possibly due to self-organization, in which the model rapidly changed states-another classic signature of complex systems. Fourth, Panels E and $\mathrm{G}$ depict oscillations between the negative and positive valence banks. Oscillations are common in complex systems.

In terms of catastrophe theory, our model exhibits some of the core qualitative properties of a catastrophic system, called "catastrophe flags,"33 eg, sudden jumps, rapid transitioning from one behavioral state to another (Figure 2, Panels $\mathrm{C}$ and $\mathrm{H})$; multimodality, having only a few pronounced behavioral states - the valence units were typically either of low activation or of an activation between 0.60 - 0.80 across most simulations; inaccessibility, having behavioral states that were unstable, eg, the valence units never rested at values other than near zero activation or between $0.60-0.80$.

\section{DISCUSSION}

The main finding of our simulation was clear. Quantum-like behavior can be displayed by a model informed by both cognitive science and the Theory of Reasoned Action. This represents an advance in quantum health behavior theory which stems directly from the work of Resnicow and Page. ${ }^{3}$ In short, the state-of-the-art in quantum health behavior now includes a theoretical compu- 
Figure 2

Time-series of Intention State for 4 Representative Simulations

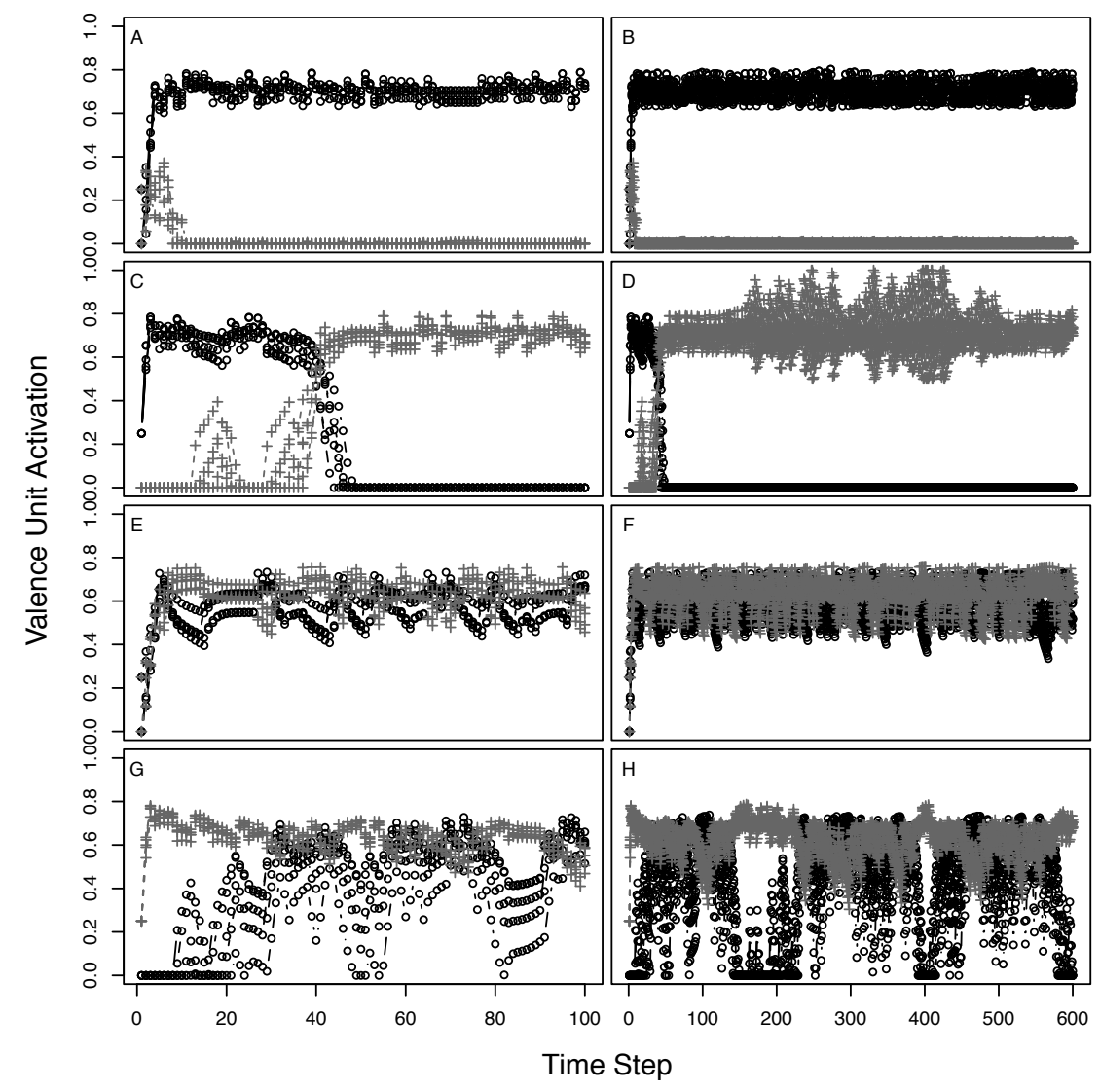

Note.

Activation values of the units in the positive valence bank are represented by the black circles; the negative valence bank by the gray crosses. Each row represents a single simulation; the first column shows the first 100 social contexts; the second shows the full simulation (600 social contexts).

tational process model.

Does this theoretical advance provide any useful insights into quantum health behavior besides proof-of-concept? The main insight is that constraint satisfaction may be the driver of quantum health behavior-ie, it is driven by the simultaneous mitigation of the current behavioral state and the immediate social context. This insight is an advance over the supposition that quantum health behavior is simply a complex system. It specifies the exact nature of the complex system, ie, constraint satisfaction, a well understood mechanism with clear behavioral properties. ${ }^{22}$ For example, a person's behavior might rapidly change states, but pinning down the cause may be difficult. Constraint satisfaction models are known to have nonlinearities that make it difficult to distinguish whether state changes arise from changing inputs (social context in our case) or from the inherent non-linearity in the constraint satisfaction mechanism.

The insight that quantum health behavior may be driven by constraint satisfaction comes with a set of methodological suggestions for further research. First, behavioral measurements should come at high temporal frequencies. Recent work in addiction has already made much progress in this area. ${ }^{25,26}$ Second, social context (or other relevant contexts) also should be measured with high temporal frequency and should be time locked to behavioral measures. Third, the idiographic approach, in which individuals are analyzed separately, is essential (see ${ }^{34}$ for a critical review of this method). In our simulations, averaging across the simulations would not have provided any insight 
into how the model behaves. We are not aware of any studies, to date, that meet these 3 requirements.

Stochastic catastrophe theory has been used successfully to analyze what are considered quantum-like behaviors in both addictive and health behaviors. ${ }^{35,36}$ This approach may prove fruitful in the analysis of future computational models of quantum behavior. However, it is important to realize that stochastic catastrophe modeling is fundamentally different from computational modeling. The former provides a statistical model, rooted in dynamical systems, of the control variables that might drive quantum behavior, whereas the latter provides a model of the actual processes that might drive quantum behavior.

There are other computational approaches in cognitive science that also may prove fruitful in the area of quantum health behavior. We explored only one class of models, constraint satisfaction models implemented in an artificial neural network. Other models such as production systems ${ }^{37}$ are of a different nature in many respects and have been applied to a wide variety of complex human behaviors (but not to health behavior). Our choice of the constraint satisfaction model stemmed from its extensive use in social psychology-the basic science that has provided a significant part of the foundation of health behavior theory.

The primary limitations of this study are threefold. First, our model was not empirically validated against human data. This limitation is part-andparcel of the natural progression of quantum health behavior theory. In the computational modeling world, it is conventional first to provide a theoretical treatment of a model and then follow up with further studies that are designed to validate (or not) the assumptions of the model empirically. ${ }^{38}$ The second limitation is that we included several assumptions in our model that are not present in the TRA. This approach is not uncommon for theoretical advances that use computational modeling. Indeed, one of the purposes of computational modeling is to make more explicit the necessary assumptions for a given phenomenon. Further research can and should begin to test the validity of our assumptions. The third limitation is that the simulation was based on the TRA and not the related TPB framework. This is an area for future work. However, given the abstract nature of our computational model, we think the results of the simulation as it stands now apply equally to the TPB.

The merger of cognitive science with health behavior theory has implications beyond quantum health behavior theory. Next, we consider some key implications of our modeling efforts for health behavior theory in general that may prove useful for evolving what we provisionally call the computational health behavior modeling approach. The primary implication is the characterization of health behavior as algorithmic.

\section{Characterization of Health Behavior as Algorithmic}

Computational health behavioral modeling is, at its core, about defining the algorithms that underlie health behavior. This is, in all respects, the overarching implication of using computational modeling to study health behavior, from which, several further implications follow.

First, the algorithmic approach provides a basis for dynamic simulation. As we illustrated, implementing quantum health behavior theory in precise algorithmic terms allowed us to see how the model behaved dynamically, over time, through the use of simulation.

Second, the computational health behavioral modeling approach distinctly separates the individual from his/her context. In short, it defines the ecological paradigm ${ }^{39}$ in algorithmic terms. It seems, at first approximation, that this approach also may inform agent-based approaches to population-level health behavior, where both the context and the individual are equally important as well as the algorithms that define their interactions.

Third, capturing health behavior as an algorithm invites comparisons among related phenomena and theory. For example, our model makes the Theory of Reasoned Action comparable to recent models of attitude formation that also use constraint satisfaction. In turn, we have at our disposal a set of new, related research questions: Is attitude formation implicit or explicit? ${ }^{40}$ and Does it invoke constructive or static memory representations ${ }^{20}$ In short, by the algorithmic approach we can see that the Theory of Reasoned Action falls into a larger theoretical arena, with new constructs and potentially new predictions that may improve our ability to intervene and prevent unhealthy behavior in populations.

Fourth, the algorthmic approach comes with a set of psychologically plausible mechanisms of learning, ${ }^{41}$ an area within health behavior research about which we know little. The Theory of Reasoned Action is a case in point. It does not address learning except to say that intention changes when beliefs change. ${ }^{42}$ In contrast, the algorithmic approach can specify exactly how the dynamics of change and learning occur, and, by extension, it may uncover potential levers for influencing what people learn. This would be useful in health promotion and interventions in which the key goal is to drive people to learn specific things about health behavior. Work using the algorithmic perspective in health behavior is only just beginning in this area. ${ }^{12}$

Fifth, the use of algorithms provides a natural link to cognitive neuroscience. The practice of progressing from an algorithmic interpretation of a phenomenon to what is considered the "physical" implementation of the algorithm, ie, neurophysiology, has been used in cognitive science for decades. ${ }^{43}$ This is of particular relevance for health behavior given that we are beginning to under- 
stand some of the neural underpinnings of related social phenomena, eg, attitude formation, ${ }^{44}$ social influence, ${ }^{45}$ social decision-making, ${ }^{46}$ and social self-regulation. ${ }^{47}$

Although speculative, we argue that this approach may eventually lead to a unique understanding of how genetics, working through neurophysiological algorithms to computational algorithms, may explain some of the variation in health behavior across a population.

\section{Conclusion}

Humans are information processing systems. Thus, health behavior fundamentally must be the result of information processing. It is not the case that the health behavior field ignores this fundamental assumption; it just has yet to capitalize on it. The computational health behavior modeling approach is an opportunity to annex health behavior theory with an algorithmic approach that may yield new benefit.

A parallel issue was raised in cognitive science, about 40 years ago, in Allen Newell's seminal paper entitled "You can't play 20 questions with nature and win: Projective comments on the papers of this symposium." ${ }^{48}$ Most of the work in cognitive science at the time was making extensive progress with the concept of information processing, making an ever increasing list of what the mind could, and could not do, in a binary, yes/no way. The mind can do $X$, over time turned into, the mind can do $X$, but only under condition $Y$, etc., etc. Newell ${ }^{48}$ argued that, although this is extremely important, it cannot ever add up to a cumulative understanding of what people really do and why; the complexity of goal-directed behavior gets missed in the process. A solution, recommended by Newell, was to build "complete process models" (p. 300) of how people actually do things - ie, computational models.

We feel that Newell's argument resonates with the state-of-the-art in health behavior change theory. Through a series of experiments, observational studies, and randomized controlled trials, the health behavior field has worked through a set of binary choices that answer the question: Did it work or not? Construct A works, but over time, it works only for certain populations. Or, construct B works for some situations, but not others, etc., etc.

What does this discussion of health behavior mean? Perhaps it means that a set of yes/no answers do not, in any respect, capture the complexity of what is dynamic about human health behavior. Similar to Newell, we are extremely impressed by the quality and progress of the health behavior field, and equally troubled by the prospect of continuing forward with business as usual.

Resnicow and Page $^{3}$ suggested that interventions might be re-conceptualized to accord with their complex adaptive systems model of quantum health behavior. Although their model was strictly metaphorical, it nonetheless suggested some interesting ideas (eg, to hit motivational levers in a periodic versus linear way). We agree with their suggestion inasmuch as they advocate for building an algorithmic model. This leaves open the challenge, naturally, of building the right model(s). The computational health behavior modeling approach aims to do this, and in the process, to go beyond metaphor into the realm of rigorous scientific explanation.

\section{Human Subjects Statement}

The work presented in this article was reviewed by the Institutional Review Board at Columbia University (Protocol Number: IRB-AAAF4243) and determined to be exempt because it does not involve any human subjects data.

\section{Conflict of Interest Statement}

The authors do not report any conflict of interest, financial or otherwise, regarding the research reported in this article.

\section{Acknowledgments}

This research was supported by the National Institutes of Health, Grant 1R21HD067570-01 (to MGO \& DCP and Peter Bearman) and an Epidemiology Merit Fellowship from the Columbia University Mailman School of Public Health (to MGO). We are grateful for Dan Romer's comments on the intricacies of the Theory of Reasoned Action.

\section{REFERENCES}

1. Baca J, Wilbourne P. Quantum change: ten years later. $J$ Clin Psychol. 2004;60(5):531-541.

2. West R, Sohal T. "Catastrophic" pathways to smoking cessation: findings from national survey. BMJ. 2006;332(7539):458-460

3. Resnicow K, Page SE. Embracing chaos and complexity: a quantum change for public health. Am J Public Health. 2008;98(8): 1382-1389.

4. Sun R. The Cambridge Handbook of Computational Psychology. New York: Cambridge Univ. Press; 2008.

5. Fishbein M, Ajzen I. Predicting and Changing Behavior: The Reasoned Action Approach. New York: Psychology Press/Taylor and Francis; 2010.

6. Fishbein M, Ajzen I. Belief, Attitude, Intention and Behaviour: An Introduction to Theory and Research. Reading, MA: Addison-Wesley; 1975.

7. Bermudez JL. Cognitive Science: An Introduction to the Science of the Mind. New York: Cambridge University Press; 2010.

8. Miller GA. The cognitive revolution: a historical review. Trends Cogn Sci. 2003;7(3):141-144.

9. Newell A, Simon HA. Computer simulation of human thinking. Science. 1961;134(3495):2011-2017.

10. Shoda Y, LeeTiernan S, Mischel W. Personality as a dynamical system: emergence of stability and distinctiveness from intra- and interpersonal interactions. Pers Soc Psychol Rev. 2002;6(4):316-325.

11. Monroe BM, Read SJ. A general connectionist model of attitude structure and change: The ACS (Attitude as Constraint Satisfaction) Model. Psychol Rev. 2008;115(3):733-759.

12. Orr MG, Thrush R, Plaut DC. The Theory of Reasoned Action as parallel constraint satisfaction: towards a dynamic computational model of health behavior. PloS One. 2013;8(5):e62409.

13. Bahr DB, Browning RC, Wyatt HR, Hill JO. Exploiting 
social networks to mitigate the obesity epidemic. Obesity. 2009;17(14):723-728.

14. Plaut DC, McClelland JL. Locating object knowledge in the brain: comment on Bower's (2009) attempt to revive the grandmother cell hypothesis. Psychol Rev. 2010;117(1):284-288.

15. Ajzen I. The theory of planned behavior. Organ Behav Hum Decis Process. 1991;50(2):179-211.

16. Fishbein M. The role of theory in HIV prevention. AIDS Care. 2000;12(3):273-278.

17. Christakis NA, Fowler JH. The collective dynamics of smoking in a large social network. $N$ Engl $J$ Med. 2008;358(21):2249-2258.

18. Galea S, Hall C, Kaplan, G. Social epidemiology and complex system dynamic modelling as applied to health behaviour and drug use research. Int $J$ Drug Policy. 2009;20(3):209-216.

19. Smith KP, Christakis NA. Social networks and health. Annu Rev Sociol. 2008;34:405-429.

20. Conrey FR, Smith ER. Attitude representation: attitudes as patterns in a distributed, connectionist representational system. Soc Cogn. 2007;25(5):718-735.

21. Shoda Y, Mischel W. Personality as a stable cognitive-affective activation network: characteristic patterns of behavior variation emerge from a stable personality structure. In Read SJ, Miller LC, (Eds). Connectionist Models of Social Reasoning and Social Behavior. Mahwah, NJ: Lawrence Erlbaum Associates; 1998:175-208.

22. Thomas M, McClelland JL. Connectionist models of cognition. In Sun R, (Eds). The Cambridge Handbook of Computational Psychology. New York: Cambridge University Press; 2008:23-58.

23. Ottati V, Krumdick ND. Attitudes and behavior: critical issues. In Ajzen I, Albarracin D, Hornik R, (Eds). Prediction and Change of Health Behavior: Applying the Reasoned Action Approach. Mahwah, NJ: Lawrence Erlbaum Associates; 2007:101-110.

24. Trafimow D. Distinctions pertaining the Fishbein and Ajzen's Theory of Reasoned Action. In Ajzen I, Albarracin D, Hornik R, (Eds). Prediction and Change of Health Behavior: Applying the Reasoned Action Approach. Mahwah, NJ: Lawrence Erlbaum Associates; 2007:23-42.

25. Warren K, Hawkins RC, Sprott JC. Substance abuse as a dynamical disease: evidence and clinical implications of nonlinearity in a time series of daily alcohol consumption. Addict Behav. 2003;28(2):369-374.

26. Zheng Y, Wiebe RP, Harrington-Cleveland $\mathrm{H}$, et al. An idiographic examination of day-to-day patterns of substance use craving, negative affect, and tobacco use among young adults in recovery. Multivariate Behav Res. 2013;48(2):241-266.

27. Mischel W, Shoda Y. A cognitive-affective system theory of personality: reconceptualizing situations, dispositions, dynamics and invariance in personality structure. Psychol Rev. 1995;102(2):246-268.

28. de Liver Y, van der Pligt J, Wigboldus D. Positive and negative associations underlying ambivalent attitudes. $J$ Exp Soc Psychol. 2007;43(2):319-326.

29. Thompson MM, Zanna MP. The conflicted individual: personality-based and domain-specific antecedents of ambivalent social attitudes. J Pers Soc Psychol. 1995;63(2):259-288.
30. Shultz TR, Lepper MR. Cognitive dissonance reduction as constraint satisfaction. Psychol Rev. 1996;103(2):219240.

31. Miller JH, Page SE. Complex Adaptive Systems: An Introduction to Computational Models of Social Life. Princeton, NJ: Princeton University Press; 2007.

32. Witkiewitz K, Marlatt GA. Modeling the complexity of post-treatment drinking: It's a rocky road to relapse. Clin Psychol Rev. 2007;27(6):724-738.

33. Gilmore R. Catastrophe Theory for Scientists and Engineers. New York: Wiley; 1981.

34. Molenaar PCM. A manifesto on psychology as idiographic science: Bringing the person back into scientific psychology, this time forever. Measurement. 2004;2(4):201-218.

35. Chen X, Lunn S, Harris C, et al. Modeling early sexual initiation among young adolescents using quantum and continuous behavior change methods: implications for HIV prevention. Nonlinear Dynamics Psychol Life Sci. 2010;14(4):491-509.

36. Clair S. A CUSP catastrophe model for adolescent alcohol use: an empirical test. Nonlinear Dynamics Psychol Life Sci. 1998;2(3):217-242.

37. Anderson JR, Lebiere C. The Atomic Components of Thought. Mahwah, NJ: Lawrence Erlbaum Associates; 1998.

38. Carley K. Validating computational models. CASOS Working Papers, Pittsburgh, PA: Carnegie Mellon University 1996. Available at: http://www.casos.cs.cmu.edu/publications/working_papers/howtoanalyze.pdf. Accessed August 1, 2013.

39. Sallis JF, Owen N, Fisher EB. Ecological models of health behavior. In Glanz K, Rimer BK, Viswanath K, (Eds). Health Behavior and Health Education. San Francisco: Wiley; 2008:465-485.

40. Gawronski B, Bodenhausen GV. Associative and propositional processes in evaluation: an integrative review of implicit and explicit attitude change. Psychol Bull. 2006;132(5):692-731.

41. Bransford JD, Brown AL, Cocking RR, et al. How People Learn: Brain, Mind, Experience and School. Washington, DC: National Academy Press; 2000.

42. Bleakley A, Hennessy M, Fishbein M, Jordan A. Using the integrative model to explain how exposure to sexual media content influences adolescent sexual behavior. Health Educ Behav. 2011;38(5):530-540.

43. Marr D. A Computational Investigation into the Human Representation and Processing of Visual Information. Cambridge, MA: MIT Press; 1982.

44. Cunningham WA, Zelazo PD. Attitudes and evaluations: a social cognitive neuroscience perspective. Trends Cogn Sci. 2007;11(3):97-104.

45. Falk EB, Way BM, Jasinska AJ. An imaging genetics approach to understanding social influence. Front Hum Neurosci. 2012;6(Article 168):1-13.

46. Rilling JK, Sanfey AG. The neuroscience of social decision-making. Annu Rev Psychol. 2011;62:23-48.

47. Heatherton TF. Neuroscience of self and self-regulation. Annu Rev Psychol. 2011;62:363-390.

48. Newell A. You can't play 20 questions with nature and win: projective comments on the papers of this symposium. In Chase WG, (Eds). Visual Information Processing. New York: Academic Press; 1973:283-308. 